crystal. The factor $\sinh \left(\frac{1}{2} g l\right)$ can be made to exceed unity, in which case we have parametric gain, i.e., image intensification. The factor $\exp \left[\frac{1}{2} i\left(\Delta \beta_{s}\right) l\right]$ in $(\mathrm{A} 22)$ is very nearly unity in most cases since $l$, in practice, will probably be limited to a few centimeters. It can easily be shown, however, that a subsequent stage of parametric mixing, which is needed to regain the image, will introduce the complimentary factor $\exp \left[-\frac{1}{2} i\left(\Delta \beta_{s}\right) l\right]$, thus correcting even this small phase deviation. The proportionality of the mixed field (27) to $E_{3}$ results, according to (A18) and (A22), in the small gain limit $g L \ll 1$.

\footnotetext{
*Work supported by the Air Force Office of Scientific Research.
}

${ }^{1}$ A. Yariv, Introduction to Optical Electronics (Holt, Rinehart, and Winston, New York, 1971).

${ }^{2}$ A. Gover, C. P. Lee, and A. Yariv, following paper, J. Opt. Soc. Am. 66, 306 (1976).

${ }^{3} \mathrm{~S}$. Kawakami and J. Nishizawa, IEE E Trans. MTT-16, 814 (1968).

${ }^{4}$ P. Baues, Opt. Electron. 1, 37 (1969).

${ }^{5} \mathrm{M}$. Born and E. Wolf, Principles of Optics, 3rd ed. (Pergamon, New York, 1965).

${ }^{6}$ See, for example, J. W. Goodman, Introduction to Fourier Optics (McGraw-Hill, New York, 1968).

${ }^{7}$ H. Kogelnik, Appl. Opt. 4, 1562 (1965).

${ }^{8}$ A. Yariv, Quantum Electronics, 2nd ed. (Wiley, New York, 1975), p. 120.

${ }^{9}$ D. Marcuse, Bell Syst. Tech. J. 52, 1169 (1973).

${ }^{10}$ E. L. Hahn, Phys. Rev. $80,580(1950)$.

\title{
Direct transmission of pictorial information in multimode optical fibers*
}

\author{
A. Gover, C. P. Lee, and A. Yariv \\ California Institute of Technology, Pasadena, California 91125 \\ (Received 21 August 1975; revised manuscript received 19 December 1975)
}

\begin{abstract}
The problem of coherent image transmission through a single multimode optical fiber is discussed. A scheme is presented for recovering the transmitted image after distortions brought about by the fiber modes dispersion. Realization of this scheme by holographic techniques and with lens systems is proposed, and its limitations pointed out. The application of this scheme in canceling out temporal signal dispersion in a multimode fiber transmission line is also discussed briefly.
\end{abstract}

\section{INTRODUCTION AND QUALITATIVE DESCRIPTION OF THE PROBLEM}

Recent developments in the area of low-loss optical fibers promise to make optical communication via fibers a reality in the near future. Most of the systems considered today, involve some sort of electrical modulation of an optical beam propagating in a fiber. The information is recovered at the receiving end after detection and decoding.

To obtain high data rates (say rates in excess of 200 Mbits/s) in the type of system described above it will be necessary to use single mode fibers in order to avoid pulse spreading or distortion due to modal dispersion. ${ }^{1}$

In this paper we wish to examine the basic problem of direct image transmission in multimode optical fibers by means of coherent light. By direct transmission we mean a system whereby an image, which, in principle, can be three dimensional, is projected onto the input end of a fiber and is recovered in real time at the output end without the need for electronic intermediaries. A second approach to the same problem based on nonlinear mixing is described in an accompanying paper. ${ }^{2}$

Let the multimode optical waveguide, henceforth to be designated as the channel, possess a discrete number $N$ of confined propagating modes of the form

$$
E_{p}(x, y, z)=\epsilon_{p}(x, y) \exp \left(-i \beta_{p} z\right),
$$

where $z$ is the cylindrical axis of the propagation channel, $\epsilon_{p}(x, y)$ is a function describing the transverse field distribution, and $\beta_{p}$ is the propagation constant of the $p$ th mode. The modes $\epsilon_{p}(x, y)$ are orthonormal in the sense

$$
\int_{\text {cross section }} \epsilon_{p}(x, y) \epsilon_{p^{\prime}}(x, y) d x d y=\delta_{p p^{\prime}} .
$$

Now consider a coherent picture field $u(x, y, 0)$ that is projected onto the input $(z=0)$ face of the channel. The field excites the discrete as well as the radiation, i. e., nonconfined, modes of the channel. Since the radiation modes are lost in a short distance and cannot contribute to the output for any reasonable channel length, we take the effective input field as

$$
u_{1}(x, y)=\sum_{p=1}^{N} A_{p} \epsilon_{p}(x, y),
$$

where, using (2),

$$
A_{p}=\int_{\text {cross section }} u(x, y, 0) \epsilon_{p}(x, y) d x d y .
$$

The field $u_{1}(x, y)$ is thus the field that results from spatial frequency band limiting of the true input field $u(x, y, 0)$. It is limited to $\sim N$ resolution elements (where $N$ is the number of confined modes), which is a reflection of the fact that the fiber has a finite numerical aperture. In the following discussion we will consider the problem of transmitting and recovering the field $u_{1}(x, y)$.

To obtain the form of field at the output end $(z=L)$ of the channel we propagate each mode with its characteristic propagation constant and receive from (3)

$$
u_{2}(x, y, L)=\sum_{p=1}^{N} A_{p} \epsilon_{p}(x, y) e^{-\alpha_{p} L} \exp \left(-i \beta_{p} L\right),
$$

where the possibility of intermode scattering has been ignored. A comparison of (5) to (3) shows that picture distortion can be attributed to two mechanisms: (a) the 
dependence of the mode loss $\alpha_{p}$ on the mode index, and (b) the modal dispersion, i.e., the dependence of the phase delay $\beta_{p} L$ on $p$.

In an ideal fiber we may have $\alpha_{p}=0$ so that distortion mechanism (a) is absent. In real fibers $\alpha_{p}$ will usually increase with $p$. The distortion due to (b), however, is of a basic nature and exists in any fiber. It is caused by the transverse confinement of the modes in the fiber which causes higher order modes to have smaller $\beta$ 's. The result is that the modes at $z=L$ are no longer in the proper phase relationship relative to each other and if $\left(\beta_{0}-\beta_{N}\right) L \gtrsim \pi$, which is the case in any practical system, the pictorial information is blurred beyond recognition.

The form of (5), however, suggests how the phase distortion can be remedied, in principle. One way would be to devise a spatial mode transformer (segregator) which localizes each mode into a unique and welldefined area. A phase correction plate with a transmission factor $\exp \left(i \beta_{p} L+i m 2 \pi\right)$ ( $m$ is some integer) is then inserted in the path of the localized mode so as to cancel the factor $\exp \left(-i \beta_{p} L\right)$ in (5). An inverse optical transformation which combines the modes back will then yield a field

$$
u_{\text {corrected }}=\sum_{p=1}^{N} A_{p} \epsilon_{p}(x, y) e^{-\alpha_{p} L},
$$

which, assuming $\alpha_{p} L \ll 1$, is an exact replica of the input field $u_{1}(x, y)$ [Eq. (3)]. The properties of the phase compensator as described above depend only on the channel and can thus be permanently affixed to it to compensate for any pictorial distortion.

The problem of image restoration described above differs from the image restoration problem described by Kogelnik and Pennington, ${ }^{3}$ who considered phase distortions as a local property of the medium. The phase correction, consequently, was applied on a point-bypoint basis. In our case the phase error is a modal property and local phase correction is ineffective. Our method applies to channels that transmit information through a set of orthogonal modes, and where the concept of local phase distortion does not apply.

Let us summarize the above discussion somewhat more precisely, referring in the process to Fig. 1. An input field

$$
u_{1}\left(x_{1}, y_{1}\right)=\sum A_{p} \epsilon_{p}\left(x_{1}, y_{1}\right)
$$

propagates through a length $L$ of an optical fiber, yielding an output field

$$
u_{2}\left(x_{2}, y_{2}\right)=\sum_{p} A_{p} \epsilon_{p}\left(x_{2}, y_{2}\right) e^{i \phi_{p}}
$$

where $\phi_{p} \equiv \beta_{p} L$. An ideal spatial mode analyzer $T$ transforms $u_{2}\left(x_{2}, y_{2}\right)$ into

$$
\tilde{u}_{2}\left(x_{3}, y_{3}\right)=T\left\{u_{2}\left(x_{2}, y_{2}\right)\right\}=\sum_{p} A_{p} e^{i \phi_{p}} \tilde{\epsilon}_{p}\left(x_{3}, y_{3}\right),
$$

where

$$
\tilde{\epsilon}_{p}\left(x_{3}, y_{3}\right) \equiv T\left\{\epsilon_{p}\left(x_{2}, y_{2}\right)\right\}
$$

is the transform field at plane $\left(x_{3}, y_{3}\right)$ owing to a single mode "input" field, $\epsilon_{p}\left(x_{2}, y_{2}\right)$ at plane $\left(x_{2}, y_{2}\right)$. The spatial segregation property of the ideal transformer $T$ is represented by the statement

$$
\tilde{\epsilon}_{p}\left(x_{3}, y_{3}\right) \tilde{\epsilon}_{p^{\prime}}\left(x_{3}, y_{3}\right)=0 \quad\left(p^{\prime} \neq p\right),
$$

i. e., the transformed modes $p$ and $p^{\prime}$ have no overlap at plane $\left(x_{3}, y_{3}\right)$ if $p \neq p^{\prime}$.

A phase corrector whose complex transmission function is $t\left(x_{3}, y_{3}\right)$ is placed in plane 3 . The correction consists of introducing a phase shift $-\phi_{p}$ throughout the domain of $\bar{\epsilon}_{p}\left(x_{3}, y_{3}\right)$. This results in an output

$$
\begin{aligned}
t\left(x_{3}, y_{3}\right) \tilde{u}_{2}\left(x_{3}, y_{3}\right) & =t\left(x_{3}, y_{3}\right) \sum_{p} A_{p} e^{i \phi_{p}} \tilde{\epsilon}_{p}\left(x_{3}, y_{3}\right) \\
& =\sum_{p} A_{p} \tilde{\epsilon}_{p}\left(x_{3}, y_{3}\right) .
\end{aligned}
$$

Next the beam propagates through an inverse transformer $T^{-1}$ that transforms the field $\tilde{\epsilon}_{p}\left(x_{3}, y_{3}\right)$ back into $\epsilon_{p}\left(x_{4}, y_{4}\right)$ at plane $\left(x_{4}, y_{4}\right)$,

$$
\begin{aligned}
u_{4}\left(x_{4}, y_{4}\right) & =T^{-1} \sum_{D} A_{p} \tilde{\epsilon}_{p}\left(x_{3}, y_{3}\right) \\
& =\sum_{D} A_{p} \epsilon_{p}\left(x_{4}, y_{4}\right)=u_{1}\left(x_{4}, y_{4}\right),
\end{aligned}
$$

i. e.; the field $u_{4}$ in plane 4 is a replica of the band limited input field $u_{1}\left(x_{1}, y_{1}\right)$.

An alternative scheme of operation may consist of

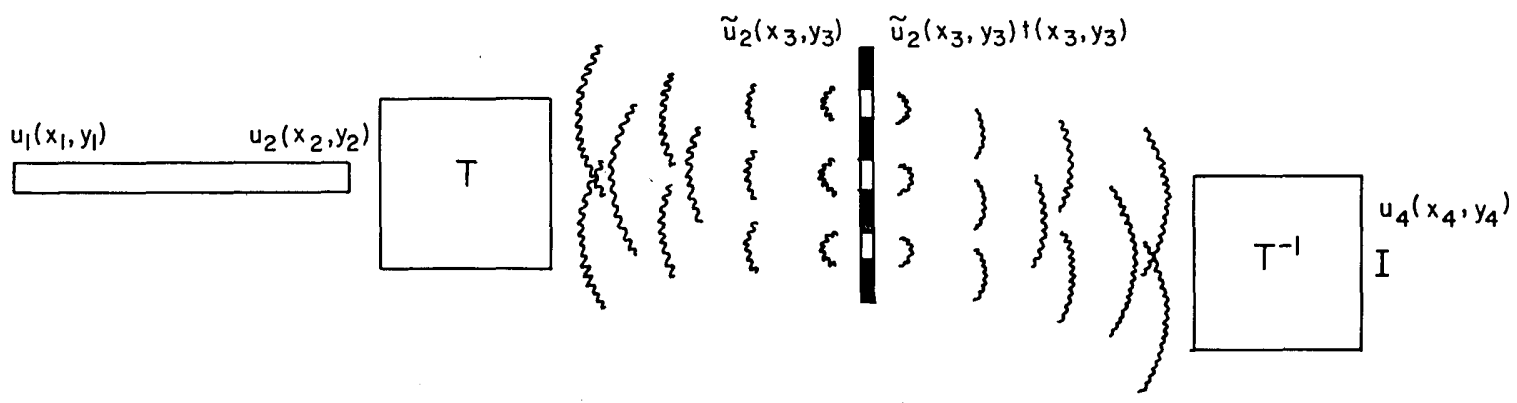

FIG. 1. Scheme for image restoration composed of (a) optical channel carrying dispersive modes; (b) optical transformer $T$ functioning as mode spatial analyser which projects each mode to an isolated spot on the transform plane $x_{3}, y_{3}$; (c) mode phase compensator plate $t\left(x_{3}, y_{3}\right)$; and (d) inverse transformer $T^{-1}$ transforming the phased mode projections from plane $x_{3}, y_{3}$ and synthesizing them on the reconstruction plane $x_{4}, y_{4}$. 


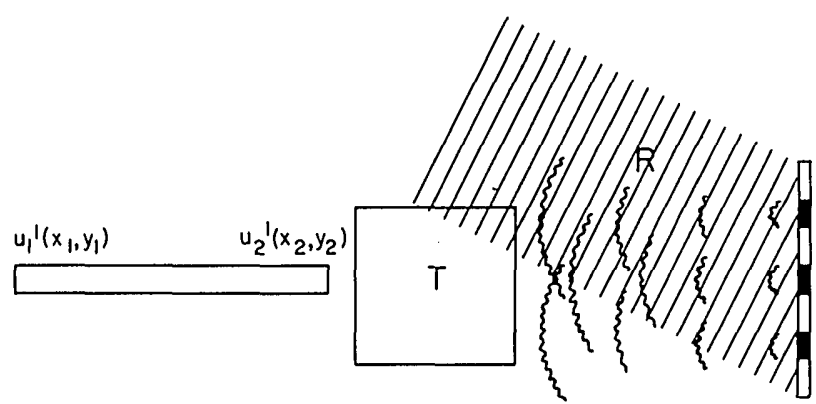

FIG. 2. Image $u_{1}^{\prime}\left(x_{1}, y_{1}\right)$ which excites all the operational channel modes, is transmitted through the channel, and its transform $(T)$ is recorded on a hologram. This hologram can be used as the phase compensator $t\left(x_{3}, y_{3}\right)$.

reversal in the scheme of Fig. 1. In this case the different modes in the fiber input are a priori appropriately phase shifted by $e^{-i \phi_{p}}$ so that the fiber dispersion will exactly cancel out these phase shifts. An undistorted image will then appear in the fiber output and no processing will be required at the receiving station!

\section{SYSTEM REALIZATION}

The scheme which was presented in Sec. I states the strategical approach to attack the problem discussed. But the practical realization of this scheme is still an open problem. A possible way to produce the optical components needed in the scheme may be provided by the developing techniques of computer generated holograms and generalized spatial filters. ${ }^{4}$ It may be possible to generate computer designed phase plates which shape the phase fronts of the incoming fields to function like the needed optical components (mode spatial analyzer and synthesizer and mode phase compensator). In this section, however, we will consider the possibility of limited image restoration with conventional means. We suggest the use of holographic techniques to produce the required phase compensator element. We discuss the limited effectiveness of a lens in producing the optical transformations required (mode segregation $T$ and mode synthesis $T^{-1}$ ) in Sec. III.

Let us assume that we have the component $T$ which is capable of performing the mode analysis transform [Eqs. (10) and (11)]. The phase compensation plate, in this case, can be produced by straightforward hologram recording techniques as demonstrated in Fig. 2. The entrance end of the fiber is illuminated with a source field $u_{1}^{\prime}\left(x_{1}, y_{1}\right)$, which excites all the modes with equal amplitude so that

$$
u_{1}^{\prime}\left(x_{1}, y_{1}\right)=\sum_{p} \epsilon_{p}\left(x_{1}, y_{1}\right) .
$$

The output field is

$$
u_{2}^{\prime}\left(x_{2}, y_{2}\right)=\sum_{p} e^{i \phi_{p}} \epsilon_{p}\left(x_{2}, y_{2}\right) \text {. }
$$

The output of the fiber $u_{2}^{\prime}\left(x_{2}, y_{2}\right)$ is projected by the spathal analyzer on a photographic fllm, and a hologram is recorded with reference beam $R\left(x_{3}, y_{3}\right)$ which originates from the same laser source. This results, after de- velopment, in a hologram with transmission function

$$
\begin{aligned}
t\left(x_{3}, y_{3}\right)= & \alpha\left(\left[\tilde{u}_{2}^{\prime}\left(x_{3}, y_{3}\right)\right] * R\left(x_{3}, y_{3}\right)\right. \\
& +\alpha \tilde{u}_{2}^{\prime}\left(x_{3}, y_{3}\right) R^{*}\left(x_{3}, y_{3}\right)+\text { dc terms },
\end{aligned}
$$

where $\alpha$ is some constant. Substituting for the reference beam $R\left(x_{3}, y_{3}\right)=\exp \left[-i\left(\beta_{x} x_{3}+\beta_{y} y_{3}\right)\right]$ (an obliquely impinging plane wave) and substituting Eq. (15) we get

$$
t\left(x_{3}, y_{3}\right)=\alpha e^{-i\left(\beta_{x} x_{3}+\beta_{y} y_{3}\right)} \sum_{p} \tilde{\epsilon}_{p}\left(x_{3}, y_{3}\right) e^{-i \phi_{p}}+\text { c.c. },
$$

where the dc term has been ignored.

The hologram $t\left(x_{3}, y_{3}\right)$ is now to be used as the phase compensator in the scheme of Fig. 1. The input field is $u_{1}\left(x_{1}, y_{1}\right)$ [Eq. (7)]. The output is $u_{2}\left(x_{2}, y_{2}\right)$ [Eq。(8)]. After the transformation, the projection field of the fiber output $\tilde{u}_{2}\left(x_{3}, y_{3}\right)$ [Eq. (9)] impinges on the hologram and is multiplied by its transmission function $t\left(x_{3}, y_{3}\right)$ [Eq. (17)],

$$
\begin{aligned}
t\left(x_{3}, y_{3}\right) \tilde{u}_{2}\left(x_{3}, y_{3}\right) & =\alpha e^{-i\left(\beta_{x} x_{3}+\beta_{y} y_{3}\right)} \sum_{p^{\prime}} \tilde{\epsilon}_{p^{\prime}} e^{-i \phi_{p}} \sum_{p} A_{p} \tilde{\epsilon}_{p} e^{i \phi_{p}} \\
& =\alpha e^{-i\left(\beta_{x} x_{3}+\beta_{y} y_{3}\right)} \sum_{p} A_{p}\left[\tilde{\epsilon}_{p}\left(x_{3}, y_{3}\right)\right]^{2},
\end{aligned}
$$

where the c.c. term in (17) has been ignored, as in conventional off-axis holography and we made use of condition (11).

Except for the coefficient, Eq. (18) looks almost like the transform of the input function equation (7) since we succeeded in eliminating the mode dispersion phasors $e^{i \phi_{p}}$. However, because the summands in Eq. (18) are $A_{p} \tilde{\epsilon}_{p}^{2}$ and not $A_{p} \tilde{\epsilon}_{p}$, an inverse transform $T^{-1}$ applied to the field of Eq. (18) (see Fig. 1) will not reproduce the input image $u_{1}\left(x_{4}, y_{4}\right)$ but a distorted form of it. A sufficient condition (which as we will see later is not a necessary condition) for avoiding this distortion is to require from the mode analyzer component not only to segregate the different mode propagation, but also to spread each mode's energy uniformly over its projection spot so that

$$
\tilde{\epsilon}_{p}\left(x_{3}, y_{3}\right) \tilde{\epsilon}_{p^{\prime}}\left(x_{3}, y_{3}\right) \approx \tilde{\epsilon}_{p}\left(x_{3}, y_{3}\right) \delta_{p p^{\prime}},
$$

instead of (11), so that we get, from (18),

$$
t \tilde{u}_{2}=\alpha e^{-i\left(\beta_{x} x_{3}+\beta_{y} y_{3}\right)} \sum_{p} A_{p} \tilde{\epsilon}_{p}\left(x_{3}, y_{3}\right) \text {. }
$$

When this field is transformed by transform $T^{-1}$ (Fig. 1) we get back the input field $u_{1}$ [Eq. (7)]. The effect of the coefficient $\exp \left[-i\left(\beta_{x} x_{3}+\beta_{y} y_{3}\right)\right]$ is identical to the effect of a grating, causing the field $\tilde{u}_{1}\left(x_{3}, y_{3}\right)$ to diffract in direction $\left(\beta_{x}, \beta_{y}\right)$ away from the hologram axis (see Fig. 1).

\section{USE OF LENSES FOR THE OPTICAL TRANSFORMS}

As we mentioned before, a lens can be used with some degree of success to perform the transforms $T$ and $T^{-1}$. A lens positioned as in Fig. 3 creates at its focal plane the Fourier transform of the field in the other focal plane ${ }^{5}$.

$T\left\{u_{2}\right\} \equiv \tilde{u}_{2}\left(x_{3}, y_{3}\right)=\left.\mathcal{F}\left\{u_{2}\left(x_{2}, y_{2}\right)\right\}\right|_{f_{x}=x_{3} / \lambda f_{1}, f_{y}=y_{3} / \lambda f_{1}}$, 


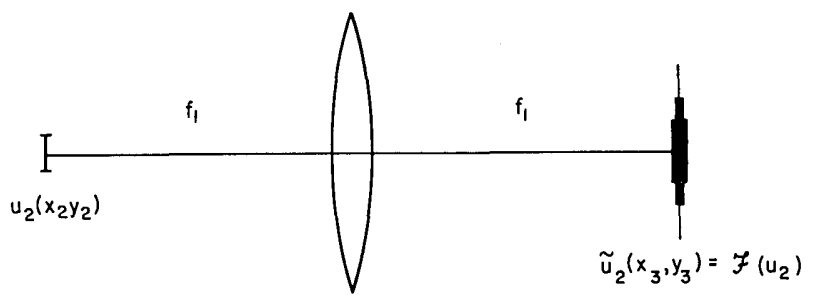

FIG. 3. Fourier transforming lens may function as the optical transformer $T$ or $T^{-1}$.

where $\lambda$ is the wavelength of the light, $f_{1}$ is the focal length, and the convention of the Fourier transform is

$$
\mathcal{F}\{u\}=\int_{-\infty}^{\infty} \int_{-\infty} u(x, y) e^{i 2 \pi\left(f_{x} x+f_{y} y\right)} d x d y .
$$

Proportionality coefficients in Eq. (21) were ignored.

A lens can also perform the inverse transform $T^{-1}$ except that the image is inverted and possibly scaled. If another lens of focal lens $f_{2}$ is placed so that its focal plane is at plane $x_{3}, y_{3}$ (Fig. 3 ) it will create the following at its other focal plane:

$$
\begin{aligned}
u_{4}\left(x_{4}, y_{4}\right) & =\left.\mathfrak{F}\left\{\tilde{u}_{2}\left(x_{3}, y_{3}\right)\right\}\right|_{f_{x}=x_{4} / \lambda f_{2}, f_{y}=x_{4} / \lambda f_{2}} \\
& =\mathfrak{F} \mathfrak{F}\left\{u_{2}\right\}=u_{2}\left(-\frac{f_{1}}{f_{2}} x_{4},-\frac{f_{1}}{f_{2}} y_{4}\right) .
\end{aligned}
$$

When a lens is used to perform the transform $T$, a somewhat different derivation of the reconstruction process can be used instead of (18). We do not require the stringent condition (19) because satisfaction of the nonoverlap condition [Eq. (11)] is sufficient. Also we do not have to require that the image $u_{1}^{\prime}\left(x_{1}, y_{1}\right)$ used to record the hologram will excite all the modes with equal amplitudes [Eq. (14)]. Rather we assume that $u_{1}^{\prime}$ $=\sum_{p^{\prime}} A_{p^{\prime}}^{\prime} \epsilon_{p^{\prime}}$. Then instead of (18) we get

$$
\begin{aligned}
t\left(x_{3}, y_{3}\right) \tilde{u}_{2}\left(x_{3}, y_{3}\right) & =\alpha e^{-i\left(\beta_{x} x_{3}+\beta_{y} y_{3}\right)} \sum_{p^{\prime}} A_{p^{\prime}}^{\prime}, \tilde{\epsilon}_{p^{\prime}} e^{-i \phi_{p^{\prime}}} \sum_{p} A_{p} \epsilon_{p} e^{i \phi_{p}} \\
& =\alpha e^{-i\left(\beta_{x} x_{3}+\beta_{y} y_{3}\right)} \sum_{p^{\prime}} A_{p^{\prime}}^{\prime} \tilde{\epsilon}_{p^{\prime}} \sum_{p} A_{p} \tilde{\epsilon}_{p} \\
& \equiv \alpha e^{-i\left(\beta_{x} x_{3}+\beta_{y} y_{3}\right)} \tilde{u}_{1}^{\prime}\left(x_{3}, y_{3}\right) \tilde{u}_{1}\left(x_{3}, y_{3}\right)
\end{aligned}
$$

where we made use of Eq. (11), only, and we did not require condition (19).

$\tilde{u}_{1}$ and $\tilde{u}_{1}^{\prime}$ are the Fourier transforms of the input image and the input image that was used for the hologram recording, respectively. These transforms were performed by a lens with focal length $f_{1}$. The inverse transform $T^{-1}$ is also a Fourier transform that is performed by another lens of focal length $f_{2}$. When the second Fourier transformation is applied to the field of Eq. (24) we can use Eq. (23) and the convolution theorem. The resulting field at the reconstruction plane $x_{4}, y_{4}$ (see Fig. 1) is

$$
\begin{aligned}
u_{4}\left(x_{4}, y_{4}\right)= & \alpha \delta\left(\frac{x_{4}}{\lambda f_{2}}-\frac{\beta_{x}}{2 \pi}, \frac{y_{4}}{\lambda f_{2}}-\frac{\beta_{y}}{2 \pi}\right) * u_{1}^{\circ}\left(-\frac{f_{1}}{f_{2}} x_{4},-\frac{f_{1}}{f_{2}} y_{4}\right) \\
& * u_{1}\left(-\frac{f_{1}}{f_{2}} x_{4},-\frac{f_{1}}{f_{2}} y_{4}\right)
\end{aligned}
$$

$$
\begin{aligned}
=\alpha u_{1}^{\prime} & \left(-\frac{f_{1}}{f_{2}} x_{4},-\frac{f_{1}}{f_{2}} y_{4}\right) \\
& * u_{1}\left(-\frac{f_{i}}{f_{2}} x_{4}+\frac{\beta_{x}}{2 \pi / \lambda} f_{1},-\frac{f_{1}}{f_{2}} y_{4}+\frac{\beta_{x}}{2 \pi / \lambda} f_{1}\right),
\end{aligned}
$$

where $*$ denotes convolution and $\delta(x, y)$ is the Dirac $\delta$ function.

Ideally, we would prefer to record the hologram with point source input image $u_{1}^{\prime}(x, y)=\delta(x, y)$. In such an ideal case Eq. (25) reduces into

$$
u_{4}\left(x_{4}, y_{4}\right)=\alpha u_{1}^{\prime}\left(-\frac{f_{1}}{f_{2}} x_{4}+\frac{\beta_{x}}{2 \pi / \lambda} f_{1},-\frac{f_{1}}{f_{2}} y_{4}+\frac{\beta_{x}}{2 \pi / \lambda} f_{1}\right) \text {. }
$$

The output field is thus an inverted, scaled (by factor $\left.f_{1} / f_{2}\right)$, and shifted replica of the original band-limited input field $u_{1}\left(x_{1}, y_{1}\right)$. In practice $u_{1}^{\prime}$ is an aperture-limited spot and its size is determined by the numerical aperture of the fiber. However, since the resolution of the input field $u_{1}(x, y)$ is also limited by the same numerical aperture, the smearing effect of the convolution in Eq. (25) will not severely degrade the resolution of the image, and Eq. (26) still holds approximately.

A key assumption used in the above analysis is that of no overlap among the transformed modes $\tilde{\epsilon}_{p}\left(x_{3}, y_{3}\right)$. This was expressed by (11) and was used in deriving Eq. (24). The need for mode separation in the transform plane can be understood physically, since it is in the transform plane that each mode undergoes the complimentary phase shift by the hologram that is needed to cancel out the dispersive phase shift accumulated by propagation in the fiber.

\section{MODE SEGREGATION BY LENSES}

At this point we should discuss the limited effectiveness of the simple lens in operating as a mode spatial analyzer as in Fig. 3. Since a lens is basically a Fourier transformer it should work well as a spatial analyzer when the modes are plane waves. We may expect a lens to function best for fibers whose modes are close to plane waves. This is the case in rectangular fibers and homogeneous circular fibers. In order to find out to what extent the lens is effective in segregating the modes we will check an example of a rectangular fiber with cross sectional dimensions $w$ and $t$.

Choosing the origin of the coordinate system at the fiber center we can approximate the transverse functions of the mode solutions by

$$
\epsilon_{m n}(x, y)=N_{m n}\left\{\begin{array}{l}
\cos \frac{m \pi x}{w} \\
\sin \frac{m \pi x}{w}
\end{array}\right\}\left\{\begin{array}{l}
\cos \frac{n \pi y}{t} \\
\sin \frac{n \pi y}{t}
\end{array}\right\} \operatorname{rect}\left(\frac{x}{w}\right) \operatorname{rect}\left(\frac{y}{t}\right),
$$

where we use the cosine functions for $m, n$ odd and the sine for $m, n$ even, $\operatorname{rect}(\xi)=1$ for $|\xi| \leq \frac{1}{2}$ and is zero elsewhere. The Fourier transform, performed by the lens [Eq. (21), Fig. 3] is

$\tilde{\epsilon}_{m n}\left(x_{3}, y_{3}\right)=\frac{N_{m n}}{i \lambda f_{1}} \frac{w t}{4}$ 


$$
\begin{aligned}
& \times\left(\frac{\sin \pi\left(t x_{3} / \lambda f_{1}-\frac{1}{2} m\right)}{\pi\left(t x_{3} / \lambda f_{1}-\frac{1}{2} m\right)} \pm \frac{\sin \pi\left(t x_{3} / \lambda f_{1}+\frac{1}{2} m\right)}{\pi\left(t x_{3} / \lambda f_{1}+\frac{1}{2} m\right)}\right) \\
& \times\left(\frac{\sin \pi\left(w y_{3} / \lambda f_{1}-\frac{1}{2} n\right)}{\pi\left(w y_{3} / \lambda f_{1}-\frac{1}{2} n\right)} \pm \frac{\sin \pi\left(w y_{3} / \lambda f_{1}+\frac{1}{2} n\right)}{\pi\left(w y_{3} / \lambda f_{1}+\frac{1}{2} n\right)}\right),
\end{aligned}
$$

where $\lambda$ is the light wavelength and $f_{1}$ the focal length.

The separation between the maxima of adjacent mode projections in, say, the $x_{3}$ direction is $\lambda f_{1} / 2 t$, while the mode width (defined as the distance from the central peak to the first zero crossing) is $\lambda f_{1} / t$. We thus have a considerable amount of overlap which will degrade the image in plane $x_{4}, y_{4}$ 。 A quantitative evaluation of this degradation has not yet been attempted.

It should be noted that the harm in mode overlap can be avoided if the image excites only a diluted group of the fiber modes so that the projections of these modes do not overlap. For example, if all the images transmitted possess reflection symmetry, they will excite only even modes. Also the hologram must be recorded in this use with even modes only! This can be suitably achieved with a point source in the center of the fiber entrance. The even mode projections have less overlap, and better image restoration may be achieved.

Even better mode segregation can be achieved with images which obtain higher symmetry properties so that they excite more diluted group of the modes (for example only every fourth mode). If symmetrization and inversion of general pictures could be performed in real time, then the scheme of Fig. 1 with holograms used for phase compensators and lenses for the transformers $T$ and $T^{-1}$ would be a practical way to restore images transmitted by a multimode fiber, in spite of the limited capability of the lens to segregate modes. The penalty that we have to pay because of this limitation is a reduction in the number of picture resolution points that can be transmitted. This reduction is proportional to the dilution of the modes and can be understood as the result of extending the size of the picture (and therefore the number of picture resolution elements) during the process of symmetry operations without increasing the information contents of the picture.

\section{IMAGE RESTORATION IN SPATIALLY INVARIANT SYSTEMS}

We would like to draw attention to the implication that the suggested scheme has to image improvement in the special class of imaging systems with spatial invariance (isoplanatic). This special case does not include optical fibers of any practical length, but includes different optical imaging systems (like telescopes, microscopes) and free space.

Suppose then that the optical channel in Figs. 1 and 2 represents a spatially invariant imaging system. The eigenmodes of a spatially invariant system are plane waves. Recalling that a (Fourier transforming) lens is an ideal spatial analyzer for plane waves, we conclude that the previously discussed scheme with lenses used for the transformers $T$ and $T^{-1}$ can be properly applied in this case for image restoration. The general analysis of the previous sections may be replaced in this case by a simpler representation.

If the impulse response function of the spatially invariant system is $h(x, y)$, so that

$$
u_{2}\left(x_{2}, y_{2}\right)=h\left(x_{2}-x_{1}, y_{2}-y_{1}\right) * u_{1}\left(x_{1}, y_{1}\right),
$$

we then get at the transform (focal) plane (Figs. 1 and 3 )

$$
\tilde{u}_{2}\left(x_{3}, y_{3}\right)=\tilde{u}_{1} \circ H \text {, }
$$

where $H$, the transfer function, and $\tilde{u}_{1}$ are the lensperformed Fourier transforms [Eq. (21)] of the impulse response function $h$ and the transmitted image $u_{1}\left(x_{1} y_{1}\right)$, respectively。

The image (which excites all plane waves) that should be used to record the hologram ( $F$ ig。2) is a point source in the origin (for which $\tilde{u}_{1}^{\prime}=1$ ). Analogously to Eq. (16) we get on the hologram transmission expression the terms $t=\alpha H^{*} R+\alpha R H^{*}$, and when this hologram is used as a spatial filter in the scheme of Fig. 1, we get after the filter from the first transmission term

$$
\begin{aligned}
\tilde{u}_{2}\left(x_{3}, y_{3}\right) t\left(x_{3}, y_{3}\right) & =\alpha|H|^{2} \tilde{u}_{1} R \\
& =\alpha\left|H\left(x_{3}, y_{3}\right)\right|^{2} \tilde{u}_{1}\left(x_{3}, y_{3}\right) e^{-i\left(\beta_{x} x_{3}+\beta_{y} y_{3}\right)} .
\end{aligned}
$$

A second Fourier transform performed on this field by the mode synthesizing lens reproduces the original image $u$, inverted, possibly scaled, and shifted in the direction $\beta_{x}, \beta_{y}$ relative to the hologram axis. The condition for this is

$$
\left|H\left(x_{3}, y_{3}\right)\right|^{2}=\text { const, }
$$

which indicates exactly the scope of possible distortions that can be recovered by the suggested scheme in spatially invariant systems.

Trivially, the condition (32) is satisfied for a perfect imaging system for which the impulse response function is

$$
h=\frac{1}{m} \delta\left(x_{2}-\frac{x_{1}}{m}, y_{2}-\frac{y_{1}}{m}\right)
$$

and the transfer function is $H=1$. However such a system of course does not need any improvement! The kind of distortions that this scheme will eliminate are those which produce transfer function

$$
H\left(f_{x}, f_{y}\right)=e^{i \phi\left(f_{x}, f_{y}\right)},
$$

which represents distortion due to phase dispersion of incoming plane waves $\left(f_{x}, f_{y}\right)$ in traversing the imaging system. This conforms with the underlying principle of the general scheme that is supposed to compensate for the phase dispersion of the transmission system eigenmodes. In this case the eigenmodes are plane waves.

The discussion of spatially invariant systems in this section does not apply to fibers and optical channels since these cannot be characterized by a transfer function (30). We find that in this particular case of spatially invariant systems the restoration scheme is closely related to the previously proposed schemes for 
correcting image distortion through an aberrating me$\operatorname{dium} .5,6$

\section{TEMPORAL SIGNAL RESTORATION}

The proposed restoration scheme can be also used to obtain temporal signal restoration in systems where pulse broadening is caused by mode dispersion $\left(e_{\circ} g_{\circ}\right.$, multimode optical fibers). The difference between this problem and the pictorial information restoration problem is that in the latter case it is sufficient to compensate the modes modulo $2 \pi$, while in the first case full compensation is required.

It follows that the scheme of Fig. 1 will work to cancel temporal dispersion only to distances such that the maximum phase shift between the modes is smaller than $2 \pi$. For a multimode fiber this distance is

$$
L<2 \pi /\left(\beta_{0}-\beta_{N}\right) \text {. }
$$

At longer distances one can use the scheme of Fig。 1 only with phase compensators that can provide phase shift of more than $2 \pi$ (by thickness modulation or other methods). Apparently, the holographically produced phase compensator described by the scheme of Fig。 2 cannot then be used.

\section{CONCLUSION}

We discussed a scheme for the restoration of images transmitted through optical fibers or any other optical transmission or imaging systems that carry the spa-. tial information by a set of orthogonal modes. These spatial modes may propagate with different phase velocity (viz., dispersive modes). The scheme consists of projecting the different modes into nonoverlapping spots, where they are appropriately phase compensated by a suitable spatial filter, and then recombining them to produce the original image. Since the scheme recreates the complex input field $m$, it is capable in principle of transmitting and restoring three-dimensional pictorial information.

In summary, holographic techniques were proposed as a possible means for performing the modes phase compensation. The application of a Fourier transforming lens for analyzing the modes and for synthesizing them back was discussed, and its limitations pointed out. Finally, we discussed briefly the application of this scheme to cancel out temporal dispersion.

*Work supported by the Air Force Office of Scientific Research.

${ }^{1}$ L. G. Cohen and S. D. Personick, Appl. Opt. 14, 1361 (1975).

${ }^{2}$ A. Yariv, preceding paper, J. Opt。 Soc. Am.66, 301 (1976).

${ }^{3}$ H. Kogelnik and $K_{0}$ S. Pennington, J。 Opt. Soc. Am. 58, 273 (1968).

${ }^{4}$ For example, O. Bryngdahl, J. Opt. Soc. Am. 64, 1092 (1.974); D. C. Chu and J. R. Fineup, Opt. Eng. 13, 189 (1974).

${ }^{5} \mathrm{~J}$. W. Goodman, Introduction to Fourier Optics (McGrawHill, New York, 1968).

${ }^{6} \mathrm{~J}$. Upatnieks, A. Vanderlugt, and E. Leith, Appl. Opt.5, 589 (1966).

\title{
Field deformation and loss caused by curvature of optical fibers
}

\author{
Dietrich Marcuse \\ Bell Laboratories, Crawford Hill Laboratory, Holmdel New Jersey 07733 \\ (Received 20 November 1975)
}

\begin{abstract}
Curvature loss formulas of optical fibers usually ignore the effect of field deformation caused by the curved axis of the fiber. Contrary to naive intuition, this field deformation may substantially decrease the radiation losses of modes with low mode number. Losses of modes with high mode numbers are, however, increased. We present a theoretical evaluation of curvature losses of the modes of a step-index fiber with a bent axis incorporating the influence of field deformation on the loss coefficient. The limitations of the simple loss formula in case of a sharply bent overmoded waveguide are pointed out.
\end{abstract}

\section{INTRODUCTION}

Formulas for the loss coefficients of the modes of a step-index fiber with bent axis have been derived by several authors. ${ }^{1-9}$ These formulas are all in essential agreement with each other, but most of them ignore the influence of field deformation that occurs as a consequence of fiber curvature. Marcatili's theory ${ }^{2}$ includes the effect of field displacement, but it does not address itself to round fibers, considering guides with rectangular cross section instead. It is usually assumed that the field in the curved fiber may be approximated by the field of the mode in the straight fiber. This approximation is reasonably accurate for the evaluation of mode losses in single-mode fibers. However, in fibers supporting many modes the fields of low-order modes may be substantially changed by the curved fiber axis, and the mode losses are altered accordingly. Naively, one might expect that the actual losses of the deformed modes should be higher than the loss prediction based on the formula that was derived by assuming that the field in the curved fiber preserves its shape at least approximately. We show in this paper that the naive assumption is wrong. The losses of the actual, deformed mode fields may be substantially lower than predicted by the simple loss formula ignoring field distortion. When the shapes of the modes in a curved fiber are plotted it becomes apparent that the mode fields not only shift away from the guide axis but also narrow substantially. It has long been known that the ability of a guided mode field to tolerate bends is dependent on the width of its field distribution. ${ }^{10,11}$ Wide fields are much more susceptible to curvature losses than narrow field distributions. In light of this observation it is not sur- 